(Aus dem Hafenkrankenhaus Hamburg.)

\title{
Ein Fall von reiner motorischer Agraphie.
}

\author{
Von \\ Dr. A. Boettiger, \\ Nervenarzt.
}

Mit 5 Textabbildungen.

(Eingegangen am \%. November 1921.)

Agraphie als Teilerscheinung einer motorischen oder sensorischen Aphasie ist ja etwas Alltägliches. Die Art der agraphischen Störungen wechselt in recht erheblicher Weise je nach der Form der aphasischen Grundstörung. Reine isolierte Agraphie ist hingegen eine seltene Beobachtung. Namentlich bei Aphasien infolge apoplektiseher Blutungen oder Erweichungen dürfte sie kaum denkbar sein. Die Hirnverletzungen der Kriegszeit mit ihrem vielfach experimentellen Charakter boten vermehrte Gelegenheit zur Erforschung isolierter Ausfallserscheinungen. Reine Alexie z. B. sah ich selbst zweimal, und sie ist auch von anderer Seite mitgeteilt worden. Eine reine Agraphie ist als Kriegsschädigung meines Wissens nie publiziert worden. Das Material unseres Krankenhauses als vorwiegendes Unfall- und Polizeikrankenhaus bietet jedoch auch in der Friedensbelegung häufig eine weitgehende Ähnlichkeit mit den Kriegsbeobachtungen. Die große Zahl der alljährlich zur Aufnahme kommenden Roheits- und Suicidfälle bringt $\mathbf{u}$. a. namentlich so manche hirnlokalisatorisch interessante Einzelheit. $\mathrm{Zu}$ diesen Raritäten gehört der Fall, den ich im folgenden mitteilen werde. Eine Schußverletzung, mit einer verhältnismäßig harmlosen Waffe und kleinem Kaliber ausgeführt, führt mit der Exaktheit eines Experiments nach Abklingen der anfänglich umfangreicheren Nachbarerscheinungen zu einer reinen motorischen Agraphie, und die Röntgenplatte gibt die Möglichkeit einer genaụen Hirnlokalisation.

Paul Fr..., 19 Jahr alt, Schiffsbauschüler, Vorgeschichte ohne Besonderheiten. Aufgenommen im Krankenhaus am 25. IV. 1921 abends 11. Uhr. Infolge Streitigkeiten in der Ingenieurschule und infolge Beschuldigungen, die ihm anseheinend nicht ganz zu Unrecht vorgeworfen wurden, hatte er abends einen Suicidversuch durch Erschießen gemacht, war bewußtlos umgefallen und sofort von der Sanitätskolonne ins Krankenhaus gebracht worden. '

2 Finger breit über dem rechten Ohrmuschelansatz eine kleine runde Finschußöffnung, starkes Hämatom der Umgebung der Wunde, der Schußkanal führt an rauhem Knochen vorbei in das Schädelinnere. 
Der junge kräftige Mann ist in gutem Emährungszustand. Gesichtsfarbe bleich. BewuBtlosigkeit resp. Sopor besteht bei der Aufnahme nicht mehr. Er jammert ununterbrochen in verworrener Weise, schreit bei der geringsten $\mathrm{B}$. rührung, teils preßt er die Augen zu, teils sieht er sich wirr um. Es besteht allgemeines Muskelzittern und motorische Unruhe. Anscheinend keine Lähmungen. Seine Sprachäußerungen sind unzusammenhängend unklar; er spricht von einer schrecklich großen Kanone. Er weiß offenbar nicht, was mit ihm vorgegangen ist.

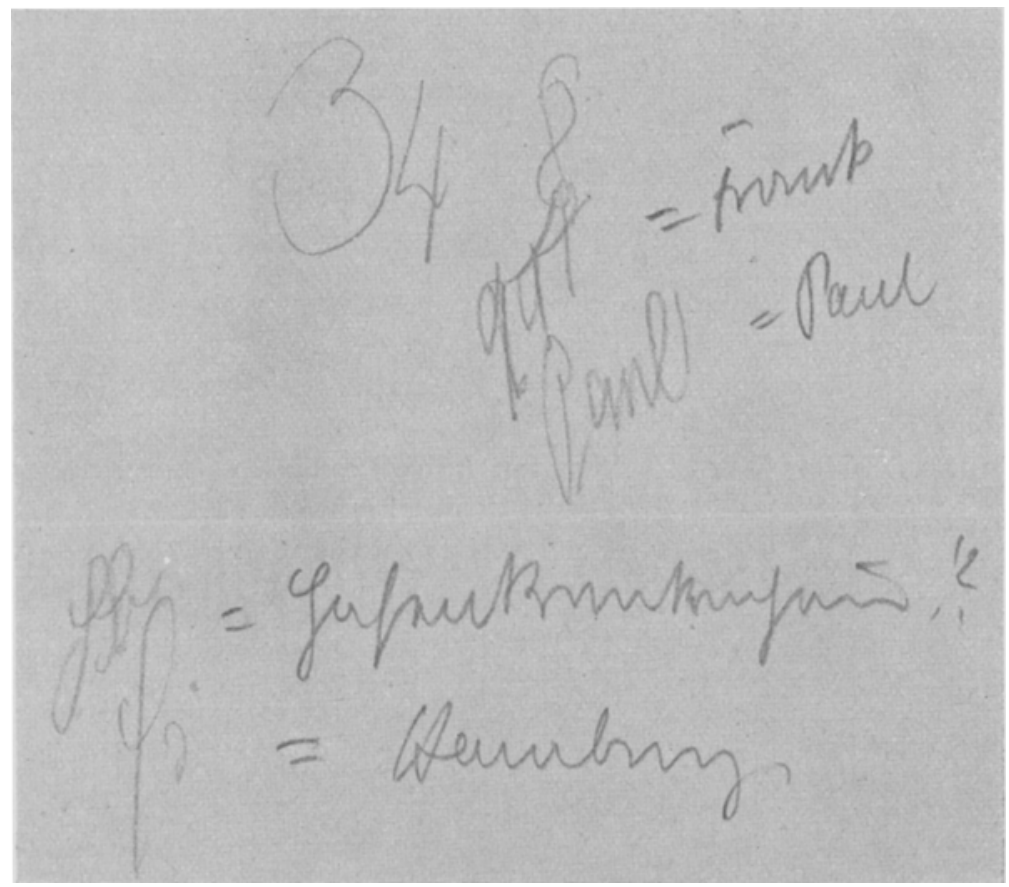

Abb. 1. a) Spontanschrift auf Diktat. Erklärung im Text.

Die körperliche Untersuchung, soweit sie möglich ist, ergibt mittelweite, gleiche Pupillen, die a uf Licht prompt reagieren, und lebhafteSehnenrefleze. Kein Brechreiz.

26. IV. Er ist sehr unruhig, stark benormmen, nimmt keine Nahrung zu sicb, greift viel nach dem Kopf, hat anscheinend heftige Kopfschmerzen. Auch am

27. IV. ist er sehr unruhig, spricht unaufbörlich zusammenhangsloses 7eug: das kaum zu verstehen ist, reagiert nicht auf Anruf, sitzt meist halb aufgerichtet auf den rechten Arm gestützt im Bett. Etwas Nackensteifigkeit. Läßt Wasser unter sich.

Am rechten Bein Oppenheim positiv.

28. IV. Lumbalpunktion: Druck $150 \mathrm{~mm}$, Liguor etwas getrübt, nicht blutig. Läßt noch unter sich.

Am 28. IV. sah ich den Kranken zuerst. Er sab halb aufgerichtet im Bett, sah ins Leere und redete mit halblauter Stimme in abgerissenen halben Sätzen vor sich hin. Von seiner Umgebung nimmt er keine Notiz. Er reagiert weder auf 
Gesichts- noch Gehörseindrücke, ist nicht zu fixieren. Nur Anfassen und Nadelstiche machen ihn unwillig. Bei Prüfung der Reflexe an den Beinen zieht er zurück und ruft zornig: "Laß das, was soll das." Die Zunge zeigt er nicht, wie er überhaupt keiner Aufforderung nachkommt. Er benimmt sich wie ein Sensorisch-Aphas i scher, vielleicht Transcortical - sensorisch - Aphasischer. Es besteht Verdacht auf eine rechtsseitige Hemianopsie. Jedoch ist ein sicheres Urteil in dieser Hinsicht nicht zu erlangen.

Der Kopf ist auf Beklopfen überall sehr empfindlich. Die Pupillen rea. gieren normal. Augenbewegungen intakt. Keine Verschiedenheit beider Faciales. Conjunctivalreflexe, überhaupt Trigeminusgebiet intakt. Der linke Arm wird spontan fast gar nicht bewegt, er reagiert auch nicht auf Nadelstiche. Dabei besteht kein motorischer Ausfall einzelner Muskelgebiete. Es ist das Bild einer Seele n lähmung des linken Arms. Motilität und Sensibilität der Beine ist in Ordnung.

Bauchdecken- und Cremasterreflexe rechts lebhafter als links, Patellarreflexe schwach, rechts schwächer als links. Achillesreflex nur links hervorzurufen. Bei Prüfung des Oppenheim links gesteigerte Plantarflexion der Zehen, rechts sehr deutlicher Oppen-

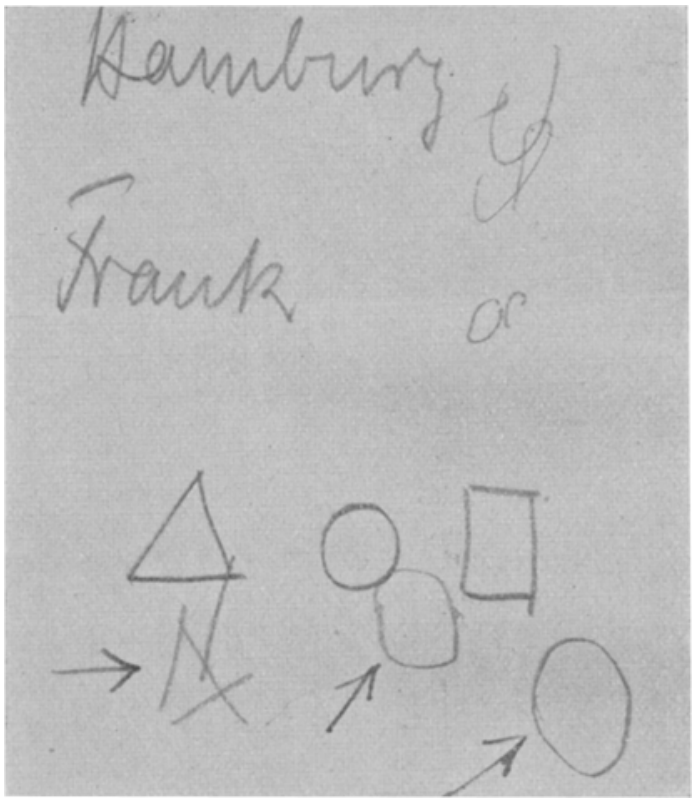

Abb. 1. b) Abschreiben.

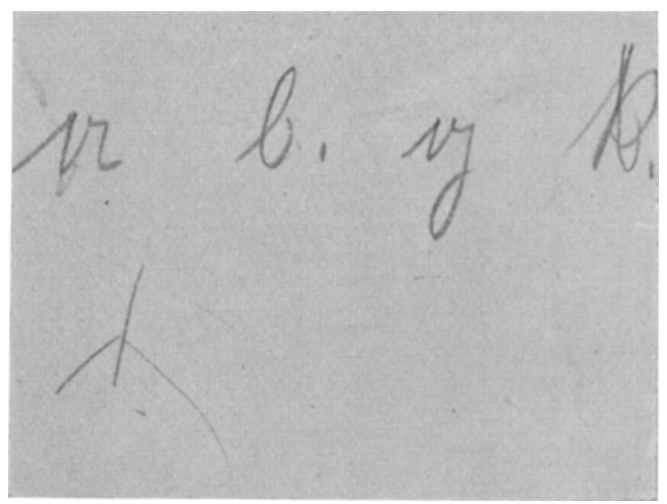

Abb. 1. c) Absehreiben.

heim. Babinski rechts wiederholt hervorzurufen, meist jedoch Dorsalflexion aller Zehen. Links zuweilen Dorsalflexion aller Zehen. Innere Organe ohne Besonderheiten.

In meinem Bericht zur Krankengeschichte schrieb ich: Der Schuß hat offenbar beim EinschuB das sensible Zentrum des linken Arms in der rechten hinteren 
Zentralwindung betroffen. Beim Auftreffen auf die Innenseite der linken Schädeldecke hat er Explosivwirkungen erzielt, die die Hirnrinde oberflächlich in Gegend des rechten Beinzentrums in der linken vorderen Zentralwindung getroffen haben (Oppenheim rechts). Da gleichzeitig Verdacht auf sensorische Aphasie besteht, muß die Flächenausbreitung der Oberflächenverletzung der linken Hemisphäre ziemlich groß angenommen werden. (Subdurales Hämatom?)

30. IV. Pat. ist etwsa klarer, besser zu fixieren. Utber Zeit und Ort ist er noch nicht orientiert. Es besteht auch deutliche retrograde Amnesie für die Zeit kurz vor dem Suicidversuch. Wenn er sich selbst überlassen ist, spricht er noeh andauernd halblaut vor sich hin, wiederholt oft die gleichen. Sätze; meist handeln sie von seinem Beruf.

Heute läßt sich bereits mit Bestimmtheit sagen, daß keine Hemianopsie, keine sensorische und motorische Aphasie mehr besteht. Der linke Arm ist noch analgetisch, Babinski besteht nicht mehr. Oppenheim ist rechts noch deutlich. Der Hirndruck ist offenbar wesentlich zurückgegangen.

3. V. Befinden noch wechselnd. Ist zeitweilig klar, zeitweilig erregt und ungeordnet.

6. V. Ganz klar, Allgemeinbefinden gut, hat keine Klagen.

9. V. Erneuter ne urologischer Status.

Seitens des Kopfes und der Extremitäten nichts Neues. Nur scheint die Analgesie des linken Armes nachzulassen. Die Sprache hat etwas Gezwungenes und Unfreies, was aber nur als Ausdruck einer gewissen psychischen Beklommenheit aufzufassen ist. Es besteht keine motorische oder sensorische Aphasie, keine Alexie. Das Lesen ist vollständig intakt. Die Untersuchung auf Sehrift gab trotzdem das überraschende Ergebnis einer totalen Agraphie. Tch ließ ihn zuerst spontan auf Diktat Zahlen schreiben. Das ging zwar mühsam und ungeschickt, aber er bekam doch wenigstens einige Zahlen aufs Papier. Dabei faßte er den Federhalter sachgemäß.

Dann ließ ich den Familiennamen Franck schreiben. Dabei zeigte sich eine deutliche Perseveration. Er schrieb einige sehr undeutliche Zahlen. Seinen Vornamen schrieb er flott; aber auch nur einmal. Später konnte er ihn nicht wiederholen. Dann diktierte ich „Hafenkrankenhaus" und „Hamburg". Abb. 1 zeigt, was dabei herauskam.

Dann schrieb ich „Hamburg" und „Frank" vor, wobei er sofort monierte, daß er sich mit ck schriebe. Nachschreiben fehlte ganz. Ich zeichnete Dreieck, Kreis und Viereck vor. Beim Nachzeichnen mußte ich immer wehren, daß er nicht in die Vorlage hinein schrieb. Das Nachzeichnen gelang gleichfalls vorbei. Endlich schrieb ich ihm einzelne Buchstaben vor, die er selbstverständlich leicht lesen lzonnte. Bereits beim a versagte er vollständig.

Nunmehr gab ich ihm den Bleistift in die linke Hand. Auch mit dieser vermochte er nichts zu schreiben.

Da sich die Störung ausnahm wie eine auf die Schrift bezügliche Apraxie, prüfte ich auch diese überhaupt. Die üblichen Prüfungsmethoden ergaben aber keine Ausfallserscheinungen. Auch eine ideatorische Apraxie fehlte. Fr konnte z. B. seine Selterwasserflasche richtig öffnen, sich Wasser einschenken und die Flasche- wieder schließen.

Zum Krankenjournal gab ich die Diagnose: Agraphie, „ob Hysterie, ob Simulation oder Lokalsymptom, bleibt vorläufig zweifelhaft".

Pat. bekam nun den Auftrag, fleißig zu üben. Er zeigte sich sehr eifrig. Das ganze Verhalten und die Art der nun schon bald einsetzenden Rückbildung der Schreiblähmung ließ den Gedanken an Hysterie sehr schnell ausschalten.

16. V. Pat. schreibt Zahlen, auch mehrstellige großenteils richtig. Vereinzelt 
neigt er dazu, Zahlen umzustellen, also anstatt 86 schreibt er 68, merkt sofort den Fehler, ist aber dann manchmal nicht imstande, die richtige Zahl dafür zu setzen. Bei Worten hat er noch große Schwierigkeiten. Er sagt selbst, ,da kommt immer etwas zwischen, und dann kann ich nicht schreiben". Immerhin gelingen schon manche kleinere Worte, er hält aber dabei noch schlecht die Linie auf dem Papier und schreibt öfter eine Linie in die andere binein.

17.V. Heute und die folgenden Tage klagt er über vermehrte Kopfschmerzen und Nackenschmerzen, kein Fieber, kein Befund. Psychisch ist er einwandfrei.

26.V. Die Sohrift hat weitere Besserung erreicht. Abb. 2 zeigt ein Diktat dieses Tages. Er sagt selbst, daß immer noch mal Worte kommen, die er nicht „durchkriegt". Auch macht er einzelne Buchstaben falsch, bringt einzelne $\mathrm{zu}$ viel, andere zu wenig. In dem beigegebenen Diktat (siehe Abbildung 2) versieht er sich bereits bei dem $\mathrm{H}$ von Hamburg. Dann will ihm das Datum nicht gelingen. Als er die 26 erst nicht sozusagen auf Anhieb fertig bekommt, kann er von derSchreibbewegung für 6 nicht herunter.

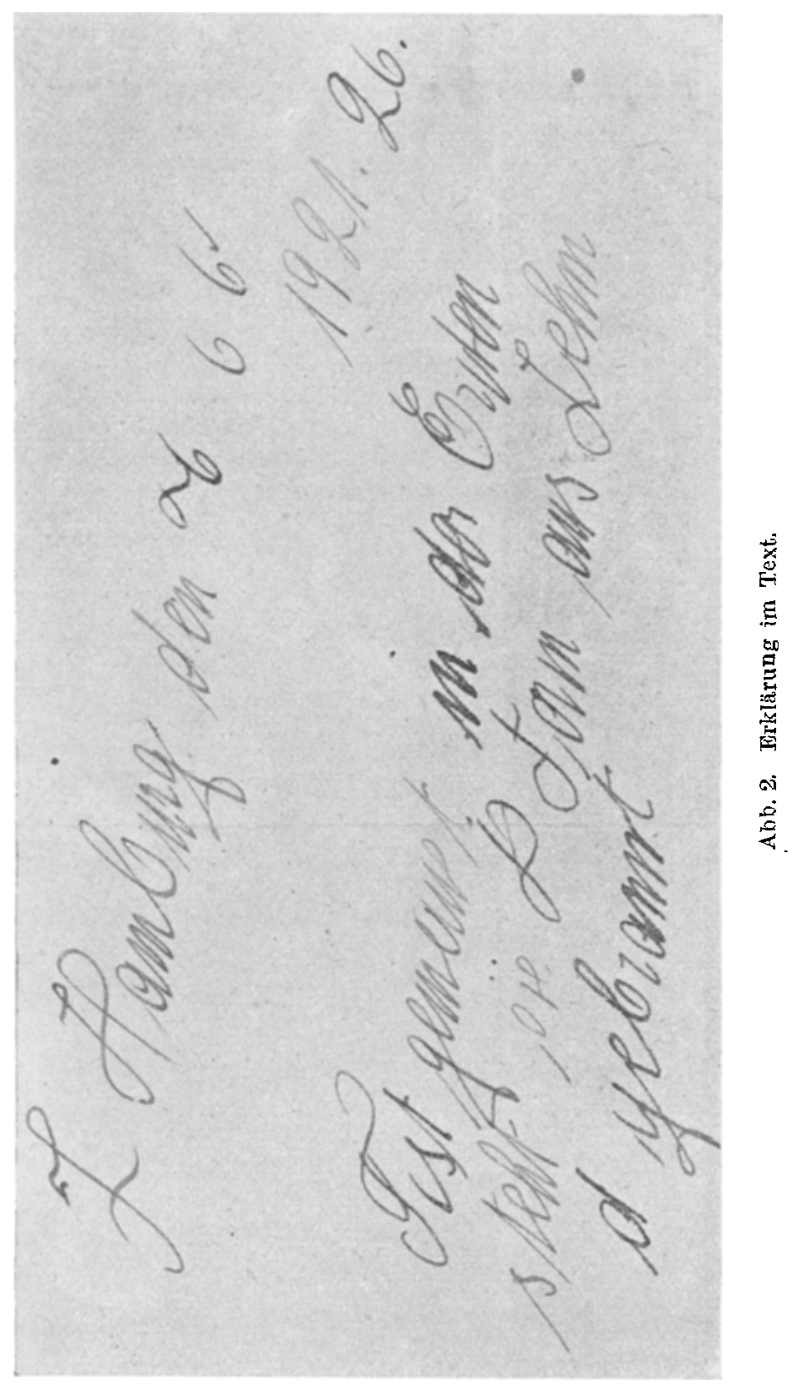
1921 schreibt er glatt, und als ich ihm darauf 26 nochmals diktierte, ging es auch. In dem Anfang von Schillers Glocke versagte er bei ,die ", "Form" und ,gebrannt". In den beiden letzten Worten war er paragraphisch.

Am 27. V. 1921 wurde er aus dem Krankenhaus entlassen.

Am 24. VI. untersuchte ich ihn wieder. Die Analgesie des linken Arms hat 
sich zurückgebildet, analgetisch ist nur noch die Beugeseite der zwei Endglieder aller Finger. Übrigens findet sich eine Andeutung der g]eichen Analgesiezone auch an der rechten Hand. An den Beinen sind die Sehnenreflexe gleich und normal. Es findet sich weder Oppenheim noch Babinski; aber rechts ist noch der Gordonsche Wadenkneifreflex im Sinne der Dorsalflexion der Großzehe positiv.

Die Schrift ist ganz wesentlich besser, auch geordneter geworden; ab und $\mathrm{zu}$ versieht er sich noch. Es ist kaum erforderlich, ausdrücklich hervorzuheben, daß er auch mit der linken Hand die normalen Schreibbewegungen jetzt machen kann, natürlich in ungeschickter Weise wie immer, wenn jemand nicht gewohnt ist, mit der linken Hand zu schreiben.

7. VII. Pat. ist jetzt so weit wiederhergestellt, daß er fast ohne Störungen schreibt; nur bei anstrengenderem hastigen Schreiben kommt es vor, daß er vereinzelt falsche Buchstaben aufs Papier bringt, die er aber sofort korrigiert. Auch beobachtet er noch zuweilen ein gewisses Häsitieren auf einzelnen Buchstaben, gewöhnlich Anfangsbuchstaben. Er ist jetzt wieder beruflich tätig. Dabei hat er aber noch eine andere Störung bemerkt, die ihm sehr peinlich ist. Die Differential- und Integralrechnung, die ihm sonst keine Schwierigkeiten machte, fällt ihm jetzt auffallend schwer. Da ich das Fach nicht beherrsche, konnte ich eine Prüfung mit dem Pat. nicht anstellen.

Nun der anatomische Befund, soweit er sich durch Röntgenaufnahmen ermitteln ließ. Die ersten Aufnahmen wurden bereits in den ersten Tagen des Krankenhausaufenthaltes gemacht und ergaben, daß das kleine Geschoß an der Innenseite des linken Os parietale abgeplattet lag. Da die Seitenaufnahme eine Übersichtsaufnahme war und der Kopf des Kranken dabei ohne genauere Einstellung auf dem Tisch gelegen hatte, ließ ich später eine neue Aufnahme machen. Dabei brachte ich die Gegend des Geschosses möglichst in den Fokus und achtete besonders darauf, daß die sagittale Mittelfläche des Kopfes möglichst parallel der Röntgenplatte und im rechten Winkel zu den auftreffenden Röntgenstrahlen zu stehen kam. Nun ergab das Bild einen ganz anderen Sitz des Geschosses wie auf dem ersten Übersichtsbild, und die Einzeichnung der Hirnwindungen in die Platte führte zu der ïberraschenden Lokaldiagnose des Sitzes der Verletzung in der Gegend des hintersten Teiles der ersten Te moralwind ung. (Abb. 3.)

Wenn wir uns jetzt den Verlauf der Verwundung und ihrer Folgeerscheinungen klinisch und anatomisch vergegenwärtigen, so kommen wir $\mathrm{zu}$ folgendem Frgebnis.

Das Geschoß durchschlug das rechte Os parietale dicht über der Schläfenschuppe, ging dann durch die hintere Zentralwindung in ihrer mittleren Höhe hindurch und traf nach Durchdringung beider Hemisphären auf die Innenfläche des linken Os parietale, wieder dicht oberhalb der Schläfenschuppe. Ein größeres Gefäß des Gehirns oder der Meningen wurde nicht verletzt. Fin intra- oder extracerebrales Hämatom trat nicht ein. Dagegen kam es zu einer partiellen reaktiven Hirnschwellung und zu Ausfallserscheinungen vox allem seitens des sensori- 
schen Sprachzentrums Wernickes. Einzelne Symptome ließen an ein anfängliches Übergreifen auch auf das Occipitalhirn und auf die Zentralwindungen denken. So bestand anfangs Verdacht auf Hemianopsie und ein deutlicher rechtsseitiger Oppenheim bei nur undeutlichem rechtsseitigen Babinski. Die Hirnschwellung ging in wenig Tagen zurück, so daß schon bald die sensorisch-aphasischen Erscheinungen schwanden. Auch die Reflexstörungen gingen bald vorüber.

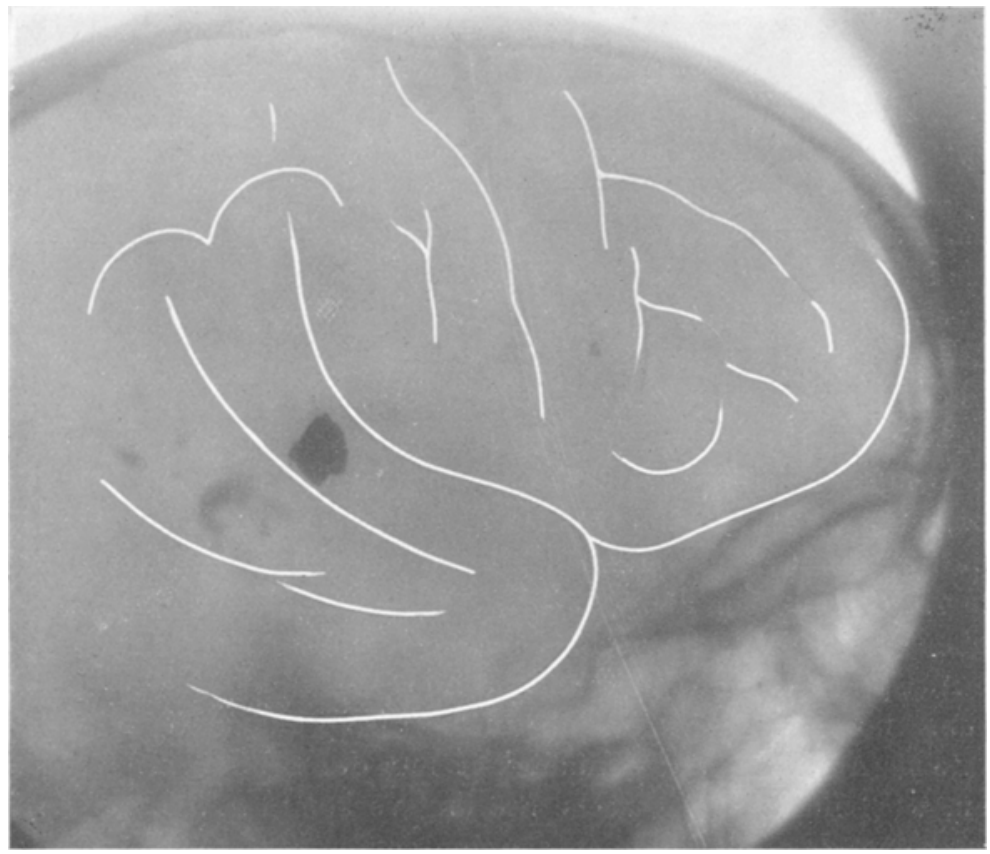

Abb. 3. Der Geschoßschatten liegt im hinteren Teile der ersten Temporalwindung. Im Gebiet der zweiten Temporalwindung einige undeutliche Schatten, die der Einschußoffinung im re $\mathbf{c h}$ t e $\mathbf{n}$ Scheitelbein entsprechen.

14 Tage nach der Verwundung ergab nun die Untersuchung, abgesehen von analgetischen Symptomen des linken Arms, von seiten der linken Hemisphäre nur noch eine reine Agraphie. Die Sehsphäre war intakt, auch bezüglich Farbensehens; die Hörsphäre war intakt, auch bezüglich der Musik; die motorische Sphäre war ohne jede Störung, es bestand auch keine Apraxie im allgemeinen Sinne dieser Anomalie. Und obwohl also alle sonstigen zur Aphasie gehörenden Symptomengruppen vollständig intakt waren, war die Agraphie, man kann wohl sagen, eine totale. Er schrieb nur einige Zahlen und einmal seinen Vornamen. Trotz vorhandener innerer und äußerer Sprache, Wortseh-, 
Wortklang- und Wortbewegungsbild waren ohne allen Ausfall, fehlte ihm vollständig die Erinnerung für die Schreibbewegungsvorstellungen. Es bestand typische Liepmannsche Apraxie, aber nur für das erlernte Schreiben.

Je isolierter eine cerebrale Störung ist, um so mehr liegt sie im Gehirn der Hirnrinde zu. Ich nehme an, daß das Zentrum für die Schreibbewegungsvorstellungen in der Rinde selbst liegt, daß es ein eigenes, streng abgegrenztes Zentrum ist.

Bezüglich der Lokalisation dieses Zentrums muß ich nur die eine Hypothese vorausschicken, die aber meiner Ansicht nach nichts Gewagtes an sich hat, nämlich, daß das Zentrum für das einzige Restsymptom, das nach Abklingen der stürmischeren Anfangserscheinungen zurückblieb, da zu suchen ist, wo das Geschoß die Rinde der linken Hemisphäre durchschlagen hat, oder mindestens in allernächster Nähe dieser Stelle. Das wäre also in unserem Falle der hinterste Teil der ersten Temporalwindung, dicht unterhalb des Gyrus supramarginalis.

Bei meiner Besprechung der einschlägigen Literatur gehe ich von der Beschreibung v. Mo nakows in seiner Gehirnpathologie, 2. Aufl. von 1905, aus, die ex der Agraphie widmet. Er führt von Fällen mehr oder weniger reiner Agraphie nur ganz wenige Autoren an, Pitres (1884), Pick, Bastian, Gordinier, Brissaud, Wernicke und Erbslöh. Im Original standen mir nur die beiden letzten zur Verfügung, beide aus dem Jahre $1903^{1}$ ). Alle diese Fälle sind untereinander durchaus ungleich und verschieden und veranlassen v. Mo nakow zu dem Ausspruch, daß die gewöhnliche Agraphie weder eine selbständige Störung noch eine solche einheitlichen Ursprungs sei. Namentlich eine reine Agraphie, also Unfähigkeit nur des Schreibens, sei höchst selten, wohl nur als hysterische Störung vorkommend. Diese Ansicht Monakows darf ich für mich als eine gewisse Entschuldigung dafür buchen, daß auch ich in meinem Falle in den ersten 2 Tagen den Verdacht einer hysterischen Störung äußerte, um ihn freilich dann sehr bald fallen zu lassen. Einen wirklichen Fall hysterischer Agraphie infolge von Zwangsvorstellungen beschreibt übrigens Donath ${ }^{2}$ ).

Auf Grund theoretischer Erwägungen und Betrachtungen teilt v. Mona kow die Fälle von Agraphie in drei Gruppen ein. Die erste Gruppe umfaßt Fälle von Agraphia cheiro-kinaesthetica (Bastian). In diesen handelt es sich um eine Apraxie mit Bezug auf die Führung der Feder. Störungen der eigentlichen sprachlichen Momente treten bei ihnen in den Hintergrund. Meist sei übrigens noch etwas $A b$ schreiben möglich, wenn auch gekritzelt. Im Hinblick auf den Fall von Pitres, dessen Patient mit der linken Hand schreiben konnte,

1) Neurol. Zentralbl. 1903, S. 968 u. 1053.

2) Dtsch. Zeitschr. f. Nervenheilk. 54, H. 1. 1915. 
ist v. Mona kow der. Ansicht, daß sich die reine cheiro-kinästhetische Agraphie nur auf die rechte Hand beziehe. Und Wernicke ${ }^{1}$ ) nimmt in seiner Besprechung des Pitresschen Falles den gleichen Standpunkt ein mit den Worten: ,Eine ganz reine Agraphie ohne alle Störung des Wortbegriffes kommt nur in der von Pitres beschriebenen, auf eine Hand beschränkten Form der Agraphie motrice pure vor, bei welcher aber gerade diese Einseitigkeit dem Begriffe der Agraphie widerspricht.“ Der eigene Fall Wernickes stellte eine fast totale Agraphie dar, die durch eine Tastlähmung der rechten Hand kompliziert war. Diese Agraphie war doppelseitig. Wernicke, der diese Form der Agraphie mit dem Namen einer literalen Form der Agraphie belegt, ist der Ansicht, daß in solchen Fällen, zu denen er außer dem eigenen auch noch die von Pick und Bastian rechnet, Vorbedingung zu ihrem Zustandekommen eine rrewisse, wenn auch unerhebliche Beeinträchtigung der inneren Sprache sei. Da diese Beeinträchtigung aber wenig in die Augen falle, könne bei oberflächlicher Betrachtung eine reine isolierte Agraphie vorgetäuscht werden. Daß diese Ansicht nicht unbe-dingt richtig ist, daß selbst bei vollständig intakter innerer Sprache eine reine doppelseitige Agraphie, und zwar die cheiro-kinästhetische Form derselben, vorkommen kann, dafür ist mein Fall ein untrüglicher Beweis.

Im Mai 1917 demonstrierte $\mathrm{Kra} \mathrm{mer}^{2}$ ) in Berlin einen Fall reiner Agraphie, der gleichfalls in diese Gruppe gehört. Bei einem 40 jährigen Luetiker trat ein Insult ein, der zu einer anfänglichen motorischen Aphasie und Apraxie und zu leichten sensorisehen Sprachstörungen führte. In wenigen Tagen gingen diese Erscheinungen ganz zurück. Es blieb eine Störung beim Lesen insofern, als Pat. die Zeilen nicht einhalten konnte, außerdem aber eine totale Agraphie. Da nun ferner noch ein eigentümliches Symptom des Vorbeigreifens und Vorbeiblickens bestand, das Kra mer auf eine gestörte räumliche Direktion der Bewegungen zurückführte, und da sich außerdem noch leise Andeutungen motorisch- und sensorisch-aphasischer Störungen nachweisen ließen, allerdings so geringe, daß sie von selbst nicht auffielen und mit Mühe gesucht werden mußten, so nahm er für die Agraphie nicht den Charakter einer isolierten Störung in Anspruch, sondern sah in ihr eine Summation gestörter sprachlicher und gestörter zentraler motorischer Funktionen. Wie aus dieser Summation freilich gerade eine reine Agraphie resultieren soll, ist mir unverständlich. Ich glaube vielmehr, daß sein Fall nicht anders aufzufassen ist als der meinige.

Es schließt sich hier noch der Fall von Erbslöh ${ }^{3}$ ) an, der allerdings

1) 1. c. und Monatsschr. f. Psychiatr. 13.

2) Neurol. Zentralbl. 1917, S. 570 .

s) $1 . \mathrm{c}$. 
nur das Bild einer partiellen Agraphie darbietet; bei ihm fehlt das Spontanschreiben ganz, während das Nachschreiben intakt ist. Hier liegt eine Schädigung der kinästhetischen Schrifterinnerungsbilder eigentlich nicht vor. Erbslöh selbst sucht den Grund für die Störung im Fehlen der optischen Schrifterinnerungsbilder. Da das Lesen aber intakt ist, scheint es sich um eine Leitungsunterbrechung zwischen zwei intakten Zentren zu handeln.

Damit sind die Fälle reiner Agraphie, soweit sie in die Gruppe 1 v. Mona kows fallen, erschöpft. Ich berichte noch kurz über die beiden arideren Gruppen, die v. Monakow aufstellt. Sie fallen gemeinsam unter Wernickes Gruppe der verbalen Agraphie, oder unter die von Heilig ${ }^{1}$ ) von der motorischen abgetrennte sensorisehe Agraphie. Als gleichsam 1. Untergruppe bezeichnet $v$. Monakow als 2. Hauptgruppe seiner Einteilung die vokale Agraphie, die nur auftritt in Verbindung mit der corticalen motorischen Aphasie und sich äußert in Lautfehlern, in der Schrift in falschen Silben und Worten. Und die 3. Hauptgruppe v. Monakows endlich sind die agraphischen Störungen bei der sensorischen Aphasie, bei der es zu Verwechslungen von. Worten, Buchstaben und Silben nach ihrer Klangverwandtsehaft kommt. In diesen beiden Gruppen ist die Technik des Schreibens ungestört. Diese beiden Gruppen sind immer nur Teilerscheinungen der entsprechenden größeren Aphasiekomplexe; eine reine Agraphie dieser Formen ist physiologisch und anatomisch undenkbar.

In meinen folgenden Erörterungen beschränke ich mich nunmehr allein auf die motorische, die cheiro-kinästhesische Form der Agraphie. Die nächstliegende Frage ist: Haben die Schreibbewegungsvorstellungen in der Hirnrinde ihr eigenes eng begrenztes Zentrum? Ist das der Fall. dann wäre ja das Zustandekommen einer reinen Agraphie ohne weiteres erklärlich. Das wird aber teilweise bestritten. Bastian hat als erster ein selbständiges cheiro-kinästhetisches Zentrum in der Fußpartie der zweiten Frontalwindung angenommen. Das wird aber von anderer Seite abgelehnt. Oppenheim²) meint,' die Utbertragung der optischen Schriftbilder geschehe wohl im motorischen Handzentrum selbst. Dieses müsse mit den Sehsphären direkt verbunden sein. Déjérine (bei Op penheim) macht dagegen das Verhalten der Schreibfähigkeit ausschließlich von dem der inneren Sprache abhängig, Störung oder Verlust der letzteren sei die Ursache der Agraphie; eine Ansicht, die nach meiner vorn mitgeteilten Kasuistik sicher falsch ist. Wernicke (1.c.) präzisiert seine patho-physiologischen Ansichten dahin, daß das optische Rindengebiet, an welches die Erinnerungsbilder der Schriftzeichen gebunden sind, zwar funktionsfähig sei, daß aber der Zusammenhang

1) Neurol. Zentralbl. 1916,' S. 964.

2) Lehrbuch, V. Aufl., S. 956. 
mit den motorischen Zentren, welche die Schreibbewegung vermitteln, verloren sei. Als geistreicher Lokalisator verlegt er den Sitz des Krankheitsherdes in eine Stelle oberhalb der hinteren Kante des linken Linsenkernes, tief im Mark des unteren Scheitellappens nahe der hinteren Zentralwindung im Fasciculus arcuatus. Hiermit sieht er also die Ursache der motorischen Agraphie offenbar in der Zerstörung einer Assoziationsbahn; ein eigenes Zentrum für die Schreibbewegungsvorstellungen scheint er nicht anzunehmen. Heilig dagegen (l. c.) betont ausdrücklich, daß es sicher Fälle gibt, wo die Funktion des Schreibens abhängig ist von der Funktion der motorischen Wortbildung und nicht allein optische Schrifterinnerungsbilder, sondern vor allem Erinnerungsbilder von Schreibbewegungen in ungestörter assoziativer Verknüpfung mit dem motorischen Handzentrum stehen müssen. Er kommt also der Idee eines eigenen Zentrums für die Schreibbewegungsvorstellungen schon einen Schritt näher.

Nicht nur mein vorn mitgeteilter Fall, sondern auch ganz allgemeine physiologische Erwägungen zwingen mich zu der Überzeugung, daß den Schreibbewegungsvorstellungen ein eigenes Zentrum zukommt. Wie allen Praxien ist auch dem erlernten Schreiben eine große Selbständigkeit eigen. Die Schreibbewegungsvorstellungen sind nicht etwa nur assoziativ eingeschaltet zwischen optisches Rindengebiet und rechte Hand. Zur Sprache gebrauchen wir stets die Sprachwerkzeuge, zum Singen Kehlkopf, Zwerchfell und obere Resonanzböden, zum Pfeifen Lippen, Gaumen und Zunge usw. Die Schreibbewegungen können wir aber nicht nur mit der rechten Hand, sondern ohne weiteres mit der linken Hand, mit den Füßen und mit dem Kopf ausführen, wenn auch nicht mit all diesen Körperteilen gleich schön. Genau entsprechend verhält es sich mit manchen anderen Praxien, worauf ich aber hier nicht näher eingehen will.

Wie die Sprache, so bedarf auch die Schreibfähigkeit einer mühsamen Erlernung. Wir brauchen uns nur an die Jahre unserer Vorschulzeit zu erinnern, wie wir jeden Buchstaben 20- und $100 \mathrm{mal}$ geübt haben, bis wir ihn geläufig hatten. Und mit Worten und Sätzen, mit Interpunktionen usw. ging es ebenso. Und wie lange blieb die Schrift schulmäßig, bis wir daran gingen, eine sogenannte ausgeschriebene Hand zu bekommen. Erlernt wird die Schrift aber nicht durch das Gehör, sondern allein durch das Sehen, also vom optischen Rindengebiet aus. Und das ist ja nur natürlich. Denn mit dem Schreiben wollen wir nichts Hörbares, sondern etwas Sichtbares reproduzieren. Daß vielleicht schon gleich beim Erlernen der Schrift die Wortklangbilder mit anklingen, ist wegen der Verwandtschaft der Schreibfähigkeit mit der Lesefähigkeit nicht verwunderlich. Das Lesen erlernen wir ja gleichzeitig durch Gesicht und Gehör. Aber das Ausüben des 
Iesens kontrollieren wir auf seine Richtigkeit mit Gesicht und Gehör, während wir die Schrift auf ihre Richtigkeit nur mit dem Gesicht kontrollieren.

Bei der Alexie sind wir längst gewohnt, ein eigenes Zentrum für die visuellen Worterinnerungsbilder als geschädigt anzusehen, und wir wissen den Sitz dieses Zentrums im Gyrus angularis. Was aber der Alexie recht ist, ist der Agraphie billig. Auch die Schreibbewegungsvorstellungen müssen ihr eigenes Zentrum haben. Nur war es bisher nicht mit Sicherheit nachweisbar.

Ich gehe aber noch weiter. Jede mühsam erlernte Fertigkeit, jede komplizierte Praxie, jede mühsam angeeignete Geschicklichkeit muß ihr Erinnerungsfeld eigens in einem Zentrum angelegt haben. Wie wäre es sonst denkbar, daß ganz isolierte Funktionsausfälle zur Beobachtung gelangten, daß dem einen nur seine Kenntnis einer fremden Sprache, dem anderen nur seine Fertigkeit im Klavierspiel, dem dritten nur seine Reproduktionsfähigkeit von Tonfolgen oder Farbenkombinationen verloren ginge. Die Kopfverletzungen des Krieges haben uns in dieser Hinsicht so manches Beispiel gebracht. Ich verweise des näheren auf die Abhandling Aschaffenburgs ${ }^{1}$ ) über ,Jokalisierte und allgemeine Ausfallerscheinungen nach Hirnverletzungen und ihre Bedeutung für die soziale Brauchbarkeit der Geschädigten", der namentlich die Wichtigkeit der Störungen der Praxie eingehend hervorhebt.

Daß das Zentrum für die Schreibbewegungsvorstellungen nicht mit einem motorischen Handzentrum zusammenfällt, geht schon allein daraus hervor, daß die Agraphie absolut nicht verbunden ist mit einer Schädigung der Motilität der Hände, weder der rechten noch der linken; ferner aber auch aus der Restitution der agraphischen Symptome. Sobald sich die Schreibbewegungsvorstellungen wieder einstellen, ist die koordinatorische Funktion der Hand- und Fingermuskeln im Sinne der Ausübung der Schrift parat und vollständig intakt. Da liegt also kein Fehler und kein Ausfall.

Ich komme nun nach diesen physiologischen Gedanken zur Anatomie, d. h. zur Hirnlokalisation. Nach v. Monakows Zusammenstellung hatte man bis dahin das Zentrum für die Schreibbewegungsvorstellungen angenommen entweder im Fuß der zweiten Frontalwindung oder im Gyrus angularis oder in der Gegend der linken oberen Temporalwindung, Wernicke in der Tiefe des unteren Scheitelläppchens im Fasciculus arcuatus. Oppenheim vermutete es im Handzentrum selbst. Einen Fingerzeig konnten uns bisher schon diejenigen Aphasiefälle geben, in denen Agraphie mit anderen aphasischen Störungen verknüpft ist. Und da zeigt sich die auffallende Tatšache, daß

1) Sammlung zwangloser Abhandl, aus dem Gebiet der Nerven- u. Geisteskrh. 11, H. 6 . 
wir die Agraphie viel häufiger mit sensorischer Aphasie vereinigt finden als mit der motorischen und gar nicht mit der subcorticalen motorischen Aphasie. Aus der großen Literatur will ich nur einige wenige Fälle aus den letzten Jahren anführen. Stier ${ }^{1}$ ) demonstriert einen Fall von isolierter Alexie und Agraphie; die letztere bezeichnet er nach Gold stein als ideatorische apraktische Agraphie. Bezüglich der Lokalisation hat er Verdacht auf den Gyrus angularis und Gyrus supramarginalis. Lewandows $\mathrm{ky}^{2}$ ) veröffentlicht einen Fall von Rechtshirnigkeit bei einem Rechtshänder. Ein großes Gliom der rechter Hemisphäre führte zu linksseitiger Hemiplegie, Hemianästhesie, Hemianopsie, sensorischer Aphasie, leichter Paraphasie, ausgeprägter Alexio und fast völliger Agraphie; dazu bestand deutliche motorische Apraxic der rechten Hand. Es fehiten motorisehe Aphasie, sowohl die corticale wie die subcorticale. In Mendels ${ }^{3}$ ) Fall einer linksseitigen Hemiplegio mit Aphasie war andererseits die Schreibfähigkeit trotz hochgradiger Zerstörung der dritten Stirnwindung und der ersten Schläfenwindung völlig erhalten, auch das Sprachverständnis intakt. Sittig ${ }^{4}$ ) beschreibt einen Fall einer Kopfverletzung in der linken Hinterhauptscheitelgegena mit leichter sensorischer Aphasie, dazu Störungen des Ziffernschreibens and des Rechnens. Multiplikation und Division fehiten ganz. $\mathbb{B}, \mathbb{E}$. Henschen $\left.{ }^{5}\right)$ berichtet in einer Arbeit ,zur Aphasie bei den otitischen Temporalabscessen ${ }^{! 6}$ über 78 Fälle. Zu einem Drittel war deütlich Worttaubheit vorhanden, in 9 Fällen gestörtes Nachsprechen, in 15 Fällen Wortblindheit, in 14 Fällen Agraphie, in 2 Fällen Jargonaphasie. In allen diesen Mitteilungen ist die Agraphie nicht so eingehend und scharf analysiert, wie ich das in meinem Falle getan habe. Der beweisendste und wichtigste aller Fälle ist der erstangeführte von Stier. Alle diese Fälle beweisen aber jedenfalls so viel, daß ebenso, wie die Erlernung der Schrift gebunden ist an benachbarte Zentren im Hinterhirn, auch offenbar die engsten räumlichen Beziehungen der Agraphiezentren bestehen $z u$ den Hinterhirnzentren und nicht zu den Vorderhirnzentren.

Nun muß ich noch einen sehr gewichtigen Autor für meine Lokalisation in Anspruch nehmen, und das ist Liepmann $\left.n^{6}\right)$. Wir haben vorn gesehen, daß die reine motorische Agraphie im Grunde genommen eine auf das Schreiben bezügliche Apraxie ist. Und Lie pmann bezeichnet als die verletzlichsten Hirngebiete quoad Praxie den ParietalJappen, dann die Zentralregion und die Parieto-Occipitalgegend.

1) Neurol. Zentralbl. 1917, S. 92.

2) Zeitschr. f. d. ges. Neurol. 4, H. 2. 1911.

3) Neurol. Zentralbl. 1914, S. 292.

4) Monatsschr. f. Psychiatr. u. Neurol. 49, 299.

5) Arch. f. Ohrenheilk. 1920. Ref. Neurol. ZentralbI. 1921, S. 208.

${ }^{-6)}$ Apraxie in Brugsch, Ergebnisse der gesamten Medizin 1920. 
Einen Sektionsbefund einer reinen Agraphie gibt es meines Wissens nicht in der Literatur. Anatomische Befunde gegen meine Lokalisation der motorischen Agraphie sind demnach nicht vorhanden. Dagegen sprechen alle die von mir angeführten klinischen Beobachtungen und die physiologischen Erfahrungen dafür, daß erstens ein begrenztes Rindenzentrum der Schreibbewegungsvorstellungen existieren muß, und zweitens, daß dieses Zentrum in der Nähe des sensorischen Sprachzentrums und des Lesezentrums liegen muß. Damit würde bewiesen sein, daß meine Hypothese, daß die Kugel die Hirnrinde der linken Hemisphäre meines Patienten da durchbohrt hat, wo die dauerhaftesten Hirnausfallserscheinungen ihren Sitz haben, keinem ernsthaften Widerstand begegnen kann. Das motorische Agraphiezentrum liegt demnach im hintersten Abschnitt der ersten Temporalwind ung, dicht unterhalb des Gyrus supramarginalis. D. h. es liegt also an der gleichen Stelle oberflächlich, wo es Wernicke in der Tiefe vermutet hatte.

Damit wäre mein Thema eigentlich erschöpft.

Aber es liegt mir noch eine Sache am Herzen, die ich vor einigen Jahren schon einmal in der Festschrift zu Oppenheims 60. Geburtstag in der D. Zeitschrift für Nervenheilkunde in einer Arbeit über Hirntumoren berührt habe. Sie betrifft die Bedeutung des Brocaschen Zentrums. Es scheint zwar seltsam, daB diese Frage mit meiner abgehandelten Arbeit in Beziehung stehen soll. Und doch ist es so. Ich hatte mich damals Marie und einigen anderen, darunter v. Niess1Me yendorf, angeschlossen in der Ansicht, daß in dem Brocaschen Zentrum im Fuße der dritten Stirnwindung nicht der Sitz für die Wortbewegungsbilder zu suchen sei. Die Zerstörung dieses Zentrums bedinge daher nicht die corticale motorische Aphasie, die habe ihren Sitz vielmehr wahrscheinlich in den Inselwindungen. Die Zerstörung der Brocaschen Windungen bedinge vielmehr das Bild der subcorticalen motorischen Aphasie, die ja gar keine Aphasie im strengen Sinne des Wortes, vielmehr eine Anarthrie sei.

- Ich habe in meinem Krankenhaus eine Reihe von Fällen zu beobachten Gelegenheit gehabt, in denen durch äußere Gewalt eine Verletzung der Broca schen Region stattgefunden hatte. Mehrfach konnte durch Trepanation die Richtigkeit der Lokaldiagnose festgestellt werden. Ich werde hoffentlich dazu kommen, die Beläge noch zu publizieren. Den jüngsten Fall will ich aber jetzt schon kurz mitteilen:

Ein Neger Rodegruiz wurde im Juni d. J. aufgenommen. Er hatte in einer Schlägerei einen Hieb über die linke Kopfseite bekommen. Die Hirnschale war eingedrückt etwa zwei Finger breit oberhalb der linken Ohrmuschel in der Gegend der Stirnbein-Scheitelbeinnaht. Durch Trepanation wurde ein Hämatom entfernt. Er hatte eine Lähmung 
der rechten Gesichtsseite und des rechten Arms, beides total, dazu eine leichte Parese des rechten Beins ohne Babinski und Oppenheim. Es bestand eine typische subcorticale motorische Aphasie, keine Spur einer Alexie oder Agraphie. Die innere Sprache war vollkommen intakt. Jeder Aufforderung $\mathrm{kam}$ er sofort nach, soweit er körperlich dazu imstande war. Mit der linken Hand schrieb er zwar ungeschickt, aber mit den richtigen Schreibbewegungen. Auch hier trotz Beteiligung der hinteren Partien der dritten und zweiten Frontalwindung kein Verlust der Wortbewegungsbilder oder der Schreibbewegungsbilder. Die Sprache hat sich bereits weitgehend wieder hergestellt; es fehlen keine Worte, aber die Sprache ist dysarthrisch. Dieses Paradigma wollen wir nun später benutzen.

Und dazu noch ein anderes aus jüngster Zait. Sorrentino ${ }^{1}$ ) teilt einen Befund mit, wo die Brocasche Windung total vernichtet war, sich trotzdem aber die Sprache wieder hergestellt hatte bis auf eine leichte Dysarthrie.

Nun muß ich zunächst etwas weiter ausholen. För tig ${ }^{2}$ ) hat einen früheren Ausspruch seines Lehrers Rieger (Úber Apparate in dem Hirn. Würzburg 1909) hervorgeholt, der mir bedeutungsvoll zu sein scheint: „Im normalen Zustand passiert alles, was durch die äußeren Sinne einläuft, zuerst den räumlichen Apparat, der hinter der Mitte des Großhirns liegt. In ihm findet Gestaltung und Objektivierung statt." Was Rieger dann weiter für Ausführungen an diesen Ausspruch knüpft, kann übergangen werden. Also was läuft durch unsere Sinne ein? Zunächst sämtliche Gesichtswahrnehmungen, die im optischen Rindenfeld ihre Engramme einlagern. Diese Gesichtswahrnehmungen erstrecken sich nun, was die Sprache anbetrifft, vor allem auf das Erkennen von Objekten und auf das Lesen. Ferner sämtliche Gehörswahrnehmungen. Dazu gehören bezüg:ich der Sprache die Wortklanghilder. Erleichtert wird ihre Aufnahme aber zweifellos, auch bei Normalhörigen, durch gleichzeitiges Ablesen von dem Gesicht des Sprechenden, also auch wieder durch Gesichtswahrnehmungen. Ihr Unterkommen findet sich im Schläfenlappen. Die Geruchs- und Geschmackswahrnehmungen, die ja in der Aphasiesymptomatik nur eine gelegentliche Rolle spielen, erwähne ich nur deshalb, weil auch ihre Lagerstätten sich hinter den Zentralwindungen befinden. Endlich die Gefühlswahrnehmungen. Hier kommen für das Gebiet der Aphasie in Frage besonders die Lagegefühls-, die Muskel- und Gelenkempfindungen, die doch zweifellos bei der Gewinnung von Schreibbewegungsvorstellungen und von Wortbewegungsbildern eine wesentliche Rolle spielen, ebenso wie bei sämtlichen Praxien. Überblicken wir die Summe

1) Neurol. Zentralbl. 1921, S. 207.

2) Uber Hirntumoren. Monatssohr. f. Neurol. u. Psyohiatr. 1921, s. 94. 
der Wahrnehmungen und der durch sie gewonnenen Vorstellungen, nicht nur, aber besonders soweit die Sprachstörungen in Betracht kommen, so finden wir alle betreffenden Zentren vereinigt hinter den Zentralwindungen, im Temporo-Parieto-Occipitalhirn. Nur die Wortbewegungsbilder werden immer noch vor die Zentralwindungen in die Brocasche Region lokalisiert. Wo wir nun jetzt die Schreibbewegungsvorstellungen nach hinten gebracht haben, dürfte der Analogieschluß berechtigt sein, daß auch der letzte Rest, die Wortbewegungsbilder, wohl hinter den Zentralwindungen unterzubringen sind. Und wenn ich nicht sehr irre, gehören die Inselwindungen phylogenetisch zum Hinterhirn. Außerdem mehren sich doch klinisch die Tatsachen, die gegen das Brocasche Zentrum als Sitz der Wortbewegungsvorstellungen sprechen, ganz erheblich.

Förtig stützt sich in seinen lokalisatorischen Erörterungen vielfach auf Philosophen und Psychologen, und zwar durchaus nicht zum Schaden unserer physiologischen Kenntnisse. Ich greife für meine Zwecke aus Kants Erkenntnistheorie den Satz heraus, daß die beiden Pole unserer Erkenntnis sind: Anschauung und Begriff. Unter Anschauung im Sinne Kants ist da zu verstehen das Feranschauen, und $d a$ er einen Sinn für alle fünf in seinem Ausdruck setzt, auch das Heranhören, Heranfüblen, Heranriechen und Heranschmecken, also alles das, was wir soeben in das Temporo-Parieto-Occipitalhirn hineinlokalisiert $r$ aben. Das Hinterhaupt dient somit der "Receptio“ im weitesten Sinne. Nun der andere Pol unserer Erkenntnis, der Begriff. Darunter versteht Kant die höhere zusammenfassende abstrakte Welt, die ,Spontaneität". Diese Seite unserer Erkenntnis wäre zu verlegen in das Stirnhirn, in das Gabiet vor den Zentralwindungen.

Ich möchte die Funktion des Stirnhirns etwas anders formulieren. Die Stirnwindungen sind der Sitz der begrifflichen und bewußt-gewollten Auswertung dessen, was wir als Anschauung im Kantschen weitesten Sinne in unseren Vorstellungsschatz aufgenommen haben. Hier sitzt bewußter Wille und Tat. Nicht umsonst liegen dicht daneben die vorderen Zentralwindungen.

Wie steht es nun mit dem Brocaschen Zentrum und mit dem Bastia n schen Zentrum im Fuße der zweiten Stirnwindung? Und jetzt komme ich zum Schluß nochmals auf Aphasie und Agraphie zurück. Beide Hirnbezirke haben auch meiner Ansicht nach mit Sprach- und Schreibfunktion zu tun. Ich supponiere aber in die Brocasche Windung ein bewuBt-koordinatorisches Zentrum für die Sprachwerkzeuge und in die zweite Stirnwindung ein bewnt-koordinatorisches Zentr um für. die Schreibmuskulatur.

Und als zweite These stelle ich die Behauptung auf, daß diese Zentren im Gegensatz zum motorischen und sensorischen Sprachzentrum nicht 
einseitig links, sondern doppelseitig angelegt und ausgebildet sind. Ist diese These richtig, dann erklärt sich leicht, warum die subcorticalmotorische Sprachstörung sich oft so überraschend schnell ausgleicht; dann ist der Befund Sorrentinos (s. vorn) und andere ähnliche erklärt. Dann ist es nicht verwunderlich, daß jeder Mensch sofort ohne Übung auch links schreiben kann, wenn auch nicht so geschickt. Dann findet der eigentümliche Fall Pitres', der eine Agraphie nur rechts hatte, seine Aufklärung dahin, daß bei ihm nicht das Schreibbewegungszentrum in der ersten Temporalwindung links, sondern das koordinatorische Zentrum in der linken zweiten Stirnwindung ausgeschaltet war.

Alle Hirnzentren sind auf Wechselwirkung aufeinander angewiesen. Gedanken ohne Inhalt sind leer, Anschauungen ohne Begriffe blind, sagt Kant. Beide Pole unserer Erkenntnis haben nur in ihrer unlösbaren Verknüpfung miteinander Bedeutung, und zwar die Bedeutung und die Manifestation des geistigen Lebens schlechthin. Die Ergründung der Lokalisationen von Einzelfunktionen des bewußten Denkens und ihre Differenzierung innerhalb des Stirnhirns ist noch ein weites Gebiet zukünftiger Forschung. 